IOS Press

\title{
Global Health
}

\section{Language and Latino immigrants living with spina bifida: Social determinants of health - the missing dimension in quality of life research}

\author{
Jonathan Castillo ${ }^{\mathrm{a}, *}$, Kellen Gandy ${ }^{\mathrm{b}}$, Viachaslau Bradko ${ }^{\mathrm{c}}$ and Heidi Castillo ${ }^{\mathrm{a}}$ \\ ${ }^{a}$ Developmental Pediatrics, Department of Pediatrics, Baylor College of Medicine, Houston, TX, USA \\ ${ }^{\mathrm{b}}$ Hematology-Oncology, Department of Pediatrics, Baylor College of Medicine, Houston, TX, USA \\ ${ }^{\mathrm{c}}$ Department of Orthopedic Surgery, Baylor College of Medicine, Houston, TX, USA
}

\begin{abstract}
.
PURPOSE: Aware of the higher birth prevalence of spina bifida (SB) among Hispanics/Latinos, we aimed to appraise the literature as it relates to cultural context through a review of quality of life (QOL) studies conducted among individuals with SB in order to improve care among immigrant families.

METHODS: A systematic review was conducted consistent with the Preferred Reporting Items for Systematic Review and MetaAnalyses (PRISMA) guidelines. The inclusion criteria were: (1) children and adolescents (5-21 years of age) with SB and/or myelomeningocele; (2) quantitative studies; (3) health-related QOL outcome measured by validated instrument determinants; and (4) US-based studies. Articles meeting inclusion criteria were assessed using the focused conceptual framework informing the study (i.e., social determinants of health).

RESULTS: Eighteen studies met inclusion criteria, with eight different QOL instruments represented. The majority of studies used generic assessments of QOL (72\%), two reported the use of both a generic and a SB-specific QOL measure (11\%), and three (17\%) documented QOL utilizing a SB-specific validated instrument. Only seven (39\%) of the studies stated that they included Hispanics/Latinos and only six (33\%) reported including Spanish-speaking individuals.

CONCLUSIONS: QOL in individuals with SB is mediated by a wide-range of interrelated factors. In order to better serve this vulnerable population as they transition across the lifespan, multilingual condition-specific QOL measures need to be further developed and implemented among Hispanic/Latino individuals with SB, especially those who are recent immigrants.
\end{abstract}

Keywords: Myelomeningocele, social determinants of health, blue marble health, health disparities, minority health, immigrant health, global health, Hispanic/Latino, systematic review

\section{Introduction}

Neural tube defects (NTDs), frequently understood as anencephaly and spina bifida (SB), have a birth

${ }^{*}$ Corresponding author: Jonathan Castillo, 8080 North Medical Drive, Suite 180, Houston, TX 77054, USA. Tel.: +1 832822 3400; Fax: +1 832825 3399; E-mail: jcporter@ texaschildrens.org. prevalence of nearly six per 10,000 live births in the United States (US) [1]. Cases arise from a combination of genetic and environmental factors that are poorly understood [2,3]. Prevalence rates of SB are also considerably influenced by the mother's race/ethnicity [4]; Hispanics/Latinos consistently have a higher birth prevalence of SB compared with the other racial/ethnic groups in the US [1]. The most common form of 
open SB is myelomeningocele (MMC), which has long been associated with numerous cognitive, mobility, urologic, and orthopedic comorbidities [5,6]. Adding to this disease burden, a number of potentially preventable complications have been described such as skin injury, infections, renal stones, and renal failure [7].

In recent years, the life expectancy of affected individuals has increased [8], and the assessment of SBrelated disease burden on their quality of life (QOL) has become progressively important in patient-centered care as they transition across the life course [9]. QOL has commonly been defined as "an individual's perception of their position in life in the context of the culture and value systems in which they live, and in relation to their goals, expectations, and concerns" [10]. The ecological framework, which recognizes that many socioeconomic influences (e.g., social gradients, stress, and social exclusion) impact and often help explain some of the divergent health outcomes found among communities, has termed these factors social determinants of health (SDH) [11,12]. This holistic approach, which elucidates multiple variables (e.g., stress and demographic variables) as contributors to the overall QOL, is not foreign to the study of QOL among individuals with SB [13].

At the same time, the US Department of Health and Human Services' Healthy People 2020 initiative underscored the importance of addressing the SDH by advocating for the creation of "social and physical environments that promote good health for all." Under this charter, social and physical determinants include: language, culture, access to health care services and schools, as well as limited exposure to deleterious social norms, physical barriers, and hazards [14]. Thus, the SDH framework encompasses four categories of interrelating factors: 1) socioeconomic circumstances, 2) psychosocial factors, 3) political, economic, and cultural drivers, and 4) neighborhood environment $[15,16]$. The Centers for Disease Control and Prevention (CDC) points to SDH as the "conditions in the environments in which people are born, live, learn, work, play, worship, and age that affect a wide range of health, functioning, and QOL outcomes and risks" [14]. The CDC has stated that when attempting to mitigate racial/ethnic health disparities, it should be remembered that SDH effectively contribute to longevity and QOL [17]. Moreover, the CDC goes on to state that healthcare delivery can be hindered if providers are not cognizant of the cultural context (e.g., language and culture); linguistic obsta- cles form a specific challenge of effectively working across cultures. Therefore, it is imperative that healthcare providers deliver support to those with language barriers to ensure care is received by all members of a community [17].

Increasingly, investigational interventions are actively pursued in an effort to treat the issues associated with SB [18,19]. As innovative procedures are subsequently applied, their impact on QOL should be studied utilizing validated instruments. In the US, in an effort to ensure that women and minorities are included in representative numbers in studies supported by the National Institutes of Health (NIH), Congress passed the NIH Revitalization Act of 1993 [20]. The directive of this act - and the ensuing redefining of sampling regulations - safeguards adequate representation of ethnic and racial minorities in research studies [21]. Concurrently, since the year 2000 there has been a $49 \%$ increase in global immigration amongst low and high-income nations [22]. In the US, $25 \%$ of children live in immigrant families [23], and of the estimated 55 million Hispanics/Latinos, nearly three of every four speaks Spanish at home while remaining the fastest-growing demographic group [24,25]. In this setting, previous systematic reviews of health-related QOL (HRQOL) assessments have called for the exploration of social environments and HRQOL [26]. We sought to examine if appropriately validated instruments, which take into account cultural context, are available and the extent of their use to assess QOL in this historically underserved US population. Therefore, the purpose of our study was to appraise the literature as it relates to language through a review of QOL studies conducted among individuals with SB in order to improve care among immigrant families.

\section{Methods}

A systematic review was conducted consistent with the Preferred Reporting Items for Systematic Review and Meta-Analyses (PRISMA) guidelines [27]. Using the MEDLINE (PubMed) and EMBASE databases, we performed a search of all articles published from 2000 to September 14, 2018, using the following terms: 'spina bifida', 'meningomyelocele', 'spinal dysraphism' AND 'quality of life', 'adaptation/physiological', 'activities/social', 'activities/physical', 'activities of daily living', 'well-being', 'personal satisfaction', 'social adjustment' in diverse combinations following $\mathrm{MeSH}$ search strategies described by McKibbon et 


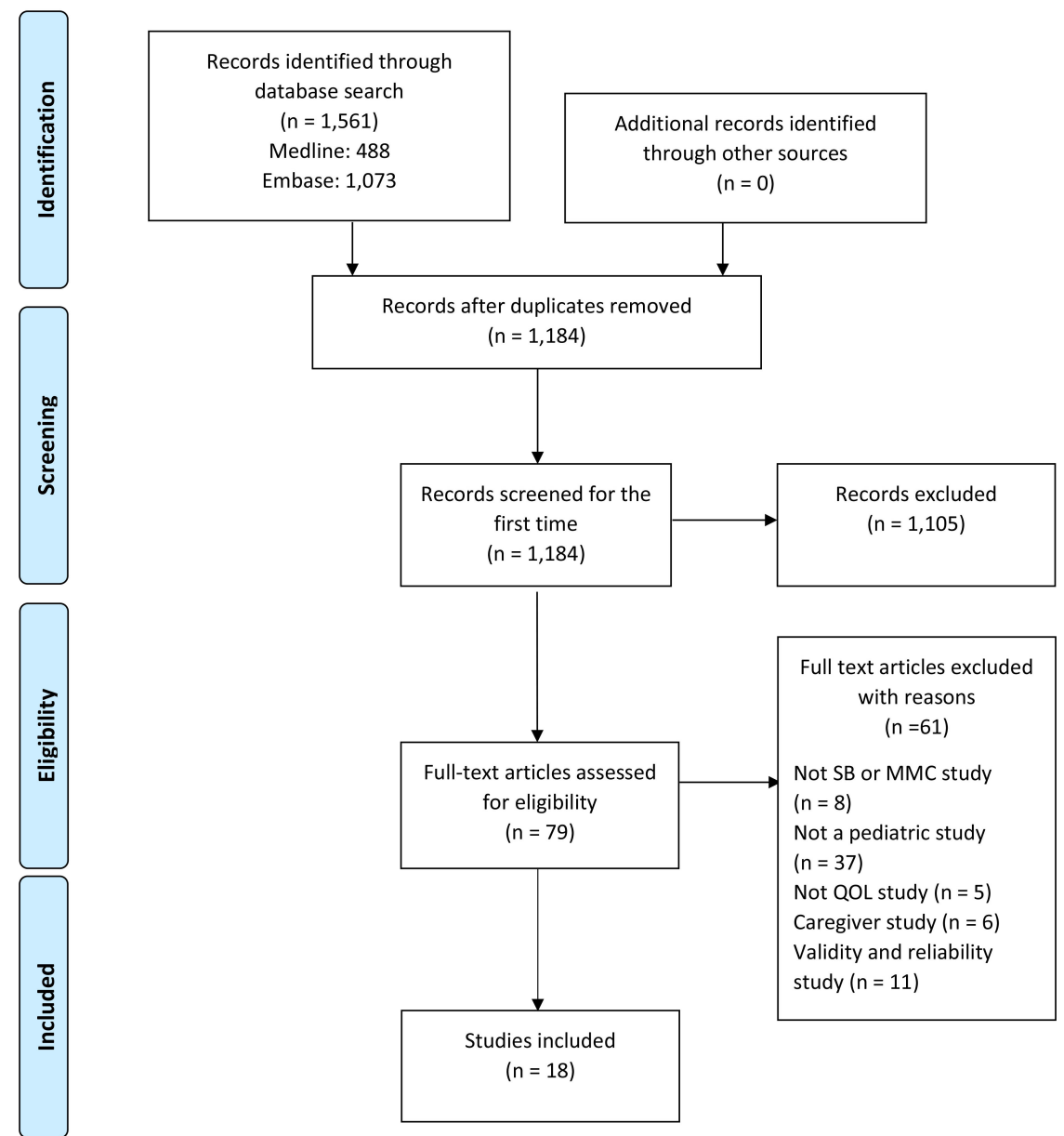

Fig. 1. PRISMA flow diagram [27]. For more information, visit www.prisma-statement.org.

al. [28]. A secondary search included examining the reference lists of all included articles; we also used Google Scholar to complete our inquiry of recently published studies. The results were restricted to English- and Spanish-language publications. The inclusion criteria were: (1) participants: children and adolescents (5-21 years of age) with SB and/or MMC; (2) quantitative studies; (3) outcome: HRQOL clearly measured by validated instrument determinants; and (4) US-based studies. Validation studies, case reports, conference abstracts, editorials, review articles, and animal studies were excluded. Studies that included fewer than six individuals with $\mathrm{SB}$ as a subset of a population also were excluded. The titles and abstracts were independently screened by two authors. After full-text articles were retrieved for eligibility, incongruences were presented to the third author, and consensus was reached through discussion. Articles meeting inclusion criteria were assessed and mapped to the focused conceptual framework informing the study (i.e., SDH). Demographic information on each study's primary catchment county (or state when applicable) was obtained from the US Census [29]. Methods of the analysis and inclusion criteria were specified in advance and documented in a review protocol which is available upon request.

\section{Results}

In accordance with PRISMA guidelines, 1,184 articles were identified, of which eighteen US-based studies met the inclusion criteria for the systematic review (Fig. 1).

\subsection{Generic vs. SB-specific QOL instruments}

In these 18 studies, eight different QOL instruments were used (Table 1). The generic (non-condition spe- 
Table 1

Summary of quality of life studies in children and adolescents with spina bifida, with annotations regarding the inclusion of cultural context

\begin{tabular}{ll}
\hline $\begin{array}{l}\text { Author (year); } \\
\text { Sample } N \text { ( } n \text { by } \\
\text { groups) age }\end{array}$ & Design/purpose/ instruments \\
\hline $\begin{array}{l}\text { Sawin et al. [13]; } \\
\text { SB } N=60 ;\end{array}$ & Correlational design to examine \\
Ages 12-21 yrs. & perceptions and factors associated \\
& with QOL measures in adolescents \\
& with SB and their parents. \\
& To examine the relationship \\
& between parent and child \\
& self-report using QOL visual \\
& analogue measures and an \\
& SB-specific QOL instrument, Teen \\
& version (i.e., SB-HRQOL).
\end{tabular}

Parekh et al. [30]; Longitudinal and cross-sectional SB $N=10$; Ages 2-18 yrs.

Abresch et
al. [31];
$N=173$ (SB
$n=42$, SCI $n=$
71, controls $n=$
60); Ages
8-20 yrs.

Cross-sectional design to compare the health-related quality of life (HRQOL) of children and adolescents with mobility impairments due to spinal cord injury (SCI) and SB to the HRQOL of children and adolescent controls without mobility impairments.

To examine the impact of mobility impairment and obesity on the HRQOL. QOL measures were collected through child self-report using a generic QOL instrument (i.e., PedsQL 4.0).

Results
Based on individual and parent reports,
there were no significant differences
between adolescents and their parents
on overall adolescent and family QOL
items. Age was related only to the
parent's perception of overall QOL,
with younger age being associated with
higher measures of QOL. There was no
significant relationship with shunt
status, level of lesion, or SB severity.
Adolescents with lower SB stress,
higher hope, positive attitudes, higher
communication efficiency, higher future
expectations, better coping skills, and
fewer sexual worries were associated
with higher QOL for self-reported
measures.

Based on individual and parent reports, the overall HRQOL was lower in children with SB than in healthy children. HRQOL reports were inconsistent between the child and the parent when assessing QOL. Six months postoperatively, children tended to self-report higher emotional and social function than did parents. QOL was not associated with clinical or demographic features, likely due to a small sample size.

Inconsistent reporting was observed between parent and child reports of QOL measures using a generic QOL instrument (i.e., PedsQL 4.0).

Based on individual-self reporting, those with SCI and SB have significantly lower QOL than those without mobility impairments. With regard to obesity, it significantly reduces QOL in children without mobility impairments but did not influence QOL in children with SCI or SB.

Nashville, TN

White 9 (90\%), Non-White $=1$ (10\%)

No language information provided. Davidson County (Nashville, TN), $N=691,243$;

White alone $=65.2 \%$, Black $=$ $27.9 \%$, American Indian $=0.5 \%$, Asian $=3.8 \%$, Pacific Islander $=$ $0.1 \%$, Two or more races $=2.5 \%$, Hispanic/Latino $=10.3 \%$, White non-Hispanic $=56.2 \%$

Davis, CA (SB only); Sacramento, CA (SCI only); Chicago, IL (SCI only) No racelethnicity information provided. No language information provided. Yolo County (Davis, CA), $N=$ 219,116;

White alone $=74.5 \%$, Black $=$ $2.9 \%$, American Indian $=1.8 \%$, Asian $=14.9 \%$, Pacific Islander $=$ $0.6 \%$, Two or more races $=5.3 \%$, Hispanic/Latino $=31.8 \%$, White non-Hispanic $=46.6 \%$ 
Table 1, continued

\begin{tabular}{|c|c|c|}
\hline $\begin{array}{l}\text { Author (year); } \\
\text { Sample } N \text { ( } n \text { by } \\
\text { groups) age }\end{array}$ & Design/purpose/ instruments & Results \\
\hline $\begin{array}{l}\text { Danielsson et } \\
\text { al. [32]; } \\
\text { MMC } N=38 \\
\text { Ages 3-16 yrs. }\end{array}$ & $\begin{array}{l}\text { Longitudinal design to investigate } \\
\text { which factors negatively influence } \\
\text { ambulatory function and explore } \\
\text { the connection among ambulation, } \\
\text { function, and HRQOL. QOL } \\
\text { measures were collected through } \\
\text { parent report using a generic QOL } \\
\text { instrument (i.e., CHQ-PF50). }\end{array}$ & $\begin{array}{l}\text { Based on parent reports, those with } \\
\text { reduced functional mobility and } \\
\text { self-care experienced lower physical } \\
\text { QOL. Individuals with MMC had } \\
\text { significantly reduced QOL compared to } \\
\text { US norms. It is important to note that a } \\
\text { generic QOL measure was used for this } \\
\text { study, and these measures are not } \\
\text { designed for individuals with physical } \\
\text { disabilities, which may explain why } \\
\text { individuals with less mobility in this } \\
\text { study reflected lower QOL scores. }\end{array}$ \\
\hline
\end{tabular}

Location/demographics/ language information/ county demographics*

Washington, DC; Baltimore, MD
White 26 (68.4\%), Non-White 11 (31.6\%)

No language information provided. Washington County (Washington, DC), $N=693,972$;

White alone $=45.1 \%$; Black $=$ $47.1 \%$; American Indian $=0.6 \%$; Asian $=4.3 \%$; Pacific Islander $=$ $0.1 \%$; Two or more races $=2.7 \%$; Hispanic/Latino $=11.0 \%$, White non-Hispanic $=36.8 \%$ Baltimore County (Baltimore,

MD), $N=832,468$; White alone $=61.4 \%$; Black $=$ 29.2\%; American Indian $=0.4 \%$; Asian $=6.4 \%$; Pacific Islander $=$ $0.1 \%$; Two or more races $=2.6 \%$; Hispanic/Latino $=5.5 \%$, White non-Hispanic $=57.2 \%$

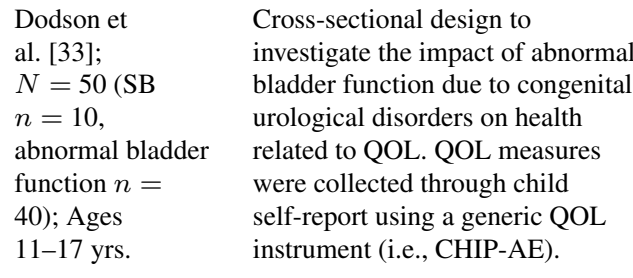

Flanagan et al. [34]; MMC $N=50$; Ages 5-18 yrs.
Cross-sectional design to compare QOL scores between children with MMC at the L2 and above motor level group and children with MMC at the L3-L5 motor level. QOL measures were collected through parent reports using a generic QOL instrument (i.e., PedsQL 4.0).

Longitudinal investigation of QOL following ACE surgery in children with SB. QOL measures were collected through parent reports using a SB-specific QOL instrument (i.e., FICQOL). Focused on surgery outcomes and on mental health (i.e., depression and anxiety) in relation to QOL.
Based on individual self-reports, adolescents with congenital urological disorders, some of which had SB, reported significantly lower QOL in the disorders domain but had comparable results to the general population for other QOL domains such as satisfaction, resilience, and achievement.

Based on parent reports, a decreased HRQOL score was observed among children with MMC at the L2 and above motor level, compared with children with MMC at the L3-L5 motor level. Moreover, the presence of a shunt was associated with a decreased HRQOL. HRQOL had no correlation with body mass index.

Based on parent reports, ACE surgery decreased the number of bowel movements and accidents, improving overall QOL. Caretaker bother, anxiety, and depression due to bowel care decreased. These results suggest that the SB-specific QOL measure that incorporates aspects of urinary and fecal incontinence may be an appropriate tool for measuring QOL outcomes in relation to surgery.

QOL instrument incorporated the individual's perception of his/her QOL.
Baltimore, MD

White 41 (82\%), Non-White 9 (18\%)

No language information provided. Baltimore County (Baltimore, MD), $N=832,468$;

White alone $=61.4 \%$; Black $=$ 29.2\%; American Indian $=0.4 \%$; Asian $=6.4 \%$; Pacific Islander $=$ $0.1 \%$; Two or more races $=2.6 \%$; Hispanic/Latino $=5.5 \%$, White non-Hispanic $=57.2 \%$

Chicago, IL

No racelethnicity information provided.

English speakers only.

Cook County (Chicago, IL), $N=$ 5,211,263;

White alone $=65.6 \%$; Black $=$ $24.0 \%$; American Indian $=0.7 \%$; Asian $=7.7 \%$; Pacific Islander $=$ $0.1 \%$; Two or more races $=1.9 \%$; Hispanic/Latino $=25.5 \%$, White non-Hispanic $=42.3 \%$

Sacramento, CA

White 13 (72\%), Non-White 5 (28\%)

No language information provided. Sacramento County (Sacramento, CA), $N=1,530,615$;

White alone $=63.4 \%$, Black $=$ $10.9 \%$, American Indian $=1.5 \%$, Asian $=16.6 \%$, Pacific Islander $=$ $1.3 \%$, Two or more races $=6.2 \%$, Hispanic/Latino $=23.3 \%$, White non-Hispanic $=44.8 \%$ 
Table 1, continued

\begin{tabular}{|c|c|c|c|}
\hline $\begin{array}{l}\text { Author (year); } \\
\text { Sample } N \text { ( } n \text { by } \\
\text { groups) age }\end{array}$ & Design/purpose/ instruments & Results & $\begin{array}{l}\text { Location/demographics/ language } \\
\text { information/ county } \\
\text { demographics* }\end{array}$ \\
\hline $\begin{array}{l}\text { Ramachandra et } \\
\text { al. [35]; } \\
N=44 \text { (shunt } \\
n=31 \text {, no shunt } \\
n=13 \text { ); Ages } \\
2-18 \text { yrs. }\end{array}$ & $\begin{array}{l}\text { Cross-sectional design to determine } \\
\text { which clinical and nonclinical (de- } \\
\text { mographic) factors influence QOL } \\
\text { in children with SB. } \\
\text { To examine the relationship } \\
\text { between parent and child } \\
\text { self-reported QOL using a } \\
\text { short-form generic QOL } \\
\text { instrument (i.e., PedsQL } 4.0 \\
\text { SF-15). }\end{array}$ & $\begin{array}{l}\text { Based on individual and parent reports, } \\
\text { those with shunted hydrocephalus (SH) } \\
\text { displayed lower self-reported physi- } \\
\text { cal health than those without a shunt. } \\
\text { However, physical health in those with } \\
\text { SH improved with age. Ethnicity, in- } \\
\text { surance, SES, ambulatory status, and } \\
\text { continence measures were not asso- } \\
\text { ciated with self-reported physical or } \\
\text { psychosocial QOL or any of the parent } \\
\text { QOL measures. } \\
\text { There was a correlation between child }\end{array}$ & $\begin{array}{l}\text { San Diego, CA } \\
\text { Hispanic } 30(68.2 \%), \text { Non- } \\
\text { Hispanic } 14(31.8 \%) \\
\text { Included Spanish speakers. } \\
\text { San Diego County (San Diego, } \\
\text { CA), } N=3,337,685 ; \\
\text { White alone }=75.6 \%, \text { Black }= \\
5.5 \%, \text { American Indian }=1.3 \%, \\
\text { Asian }=12.5 \% \text {, Pacific Islander }= \\
0.6 \%, \text { Two or more races }=4.4 \%, \\
\text { Hispanic/Latino }=33.9 \%, \text { White } \\
\text { non-Hispanic }=45.5 \%\end{array}$ \\
\hline
\end{tabular}

Flanagan et al. [36]; $N=137$ (SCI $n=83$, SB $n=$ 54); Ages $1-18$ yrs.

Freeman et al. [43]; SB $N=104$; Ages 5-12 yrs.
Cross-sectional design to describe psychosocial outcomes of children and adolescents with early-onset SCI and SB and identify differences between them. QOL measures were collected through child self-reports using a generic QOL instrument (i.e., PedsQL 4.0). To describe and compare HRQOL, participation, and mental health (i.e., depression and anxiety) outcomes.

Cross-sectional design to evaluate the relationship between child and parent reported QOL and bowel and bladder continence among young children with SB using both a generic (PedsQL 4.0) and SB-specific QOL instrument (SB-HRQOL a.k.a. QOLSBQ). self-report and parent report on psychosocial measures, but no correlation between physical health scores.

Based on individual self-reports, participants with SCI reported higher school QOL and lower anxiety with social concerns or concentration. SCI individuals participated in more activities at school than did participants with SB and trended toward fewer depressive symptoms.

Based on individual and parent reports, child and parent QOL scores were positively correlated on the generic PedsQL 4.0. Parents rated children's QOL lower than children's self-report. QOL scores did not differ based on continence status. Total PedsQL 4.0 scores were associated with age and mobility based on child's report and with mobility based on parent's report. Both generic and SB-specific instruments were used for measuring QOL in SB. SB-specific QOL instrument incorporated the individual's perception of QOL via parent-report by asking questions related to personal relationships, internal beliefs, independence, and future goals.
Chicago, IL (SB only) Philadelphia, PA; Sacramento, CA

$S B$ participants $=54-$ White 38 (70.4\%), Black 7 (13\%), American Indian 0 (0\%), Spanish Origin 6 (11.1\%), Asian 1 (1.9\%), Unclassified $1(1.9 \%)$

English speakers only in SB group. Cook County (Chicago, IL), $N=$ 5,211,263;

White alone $=65.6 \%$; Black $=$ 24.0\%; American Indian $=0.7 \%$; Asian $=7.7 \%$; Pacific Islander $=$ $0.1 \%$; Two or more races $=1.9 \%$; Hispanic/Latino $=25.5 \%$; White non-Hispanic $=42.3 \%$

Los Angeles, CA (site with Spanish speakers); Portland, OR; Seattle, WA

No racelethnicity information provided.

Included Spanish speakers.

Los Angeles County (Los Angeles, CA), $N=10,163,507$;

White $=70.9 \%$, Black $=9.0 \%$, American Indian $=1.4 \%$, Asian $=$ $15.3 \%$, Pacific Islander $=0.4 \%$, Two or more races $=3.0 \%$, Hispanic/Latino $=48.6 \%$, White non-Hispanic $=26.2 \%$ 
Table 1, continued

\begin{tabular}{lll}
\hline $\begin{array}{l}\text { Author (year); } \\
\text { Sample } N \text { ( } n \text { by } \\
\text { groups) age }\end{array}$ & Design/purpose/ instruments & Res \\
\hline $\begin{array}{l}\text { Strine et al. [37]; } \\
N=85 \text { (SB }\end{array}$ & Cross-sectional design to evaluate & health-related benefit in children \\
$n=48$, other $=$ & undergoing surgical reconstruction & i \\
37); Ages 6 mo. - & for urinary and fecal incontinence \\
14 yrs. & from a parental perspective. QOL \\
& measures were collected through \\
& parent reports using a generic QOL \\
& instrument (i.e., Glasgow \\
& Children's Benefit Inventory). \\
& Focused on surgery outcomes in \\
& relation to QOL.
\end{tabular}

Murray et

al. [38];

SB $N=134$

(time 1), $S B$

$N=109$ (time

2); Ages

8-17 yrs.
Results

Based on parent reports, moderate

improvements in parental report of

HRQOL in children undergoing

surgical reconstruction for urinary and fecal incontinence.

Longitudinal examination of QOL in children and adolescents with SB.

To examine the relationship between parent and child-self reports using a generic $\mathrm{QOL}$ instrument (i.e., PedsQL 4.0).

Rocque et

al. [39];

$N=159$ (MMC

$n=125$,

lipomyelomen-

ingocele $n=25$,

other $n=9$ );

Ages 5-20 yrs.
Cross-sectional design to explore various aspects of HRQOL in children with spinal dysraphism. QOL measures were collected through parent and/or child self-reports using a generic QOL instrument (i.e., HUI-3).
Based on individual and parent reports, children with SB display reduced physical HRQOL compared to nonclinical and other chronic health condition populations. Parent and youth self-reports were inconsistent, with youth reporting higher levels of physical and social QOL than did parents.

Most parent and child self-reports domains remained stable over time. However, longitudinal improvements in social QOL were observed only among youth self-report.

Based on individual and/or parent reports, those with MMC had lower HRQOL scores compared to those with closed spinal dysraphism. Individuals with impaired bowel continence had lower overall QOL. History of ventriculoperitoneal shunt and Chiari II decompression correlated with lower QOL scores.

Based on individual and parent reports, children tended to report higher QOL than parent reported on physical, emotional, and social functioning domains. Positive well-being as measured by the subject exercise experience scale was significantly related to overall QOL. Inconsistent reporting was observed between parent and child reports of QOL measures using a generic $\mathrm{QOL}$ instrument (i.e., PedsQL 4.0).
Location/demographics/ language information/ county demographics*

Indianapolis, IN

No racelethnicity information provided.

No language information provided. Marion County (Indianapolis, IN), $N=950,082$;

White alone $=64.6 \%$, Black $=$ $28.6 \%$, American Indian $=0.5 \%$, Asian $=3.4 \%$, Pacific Islander $=$ $0.1 \%$, Two or more races $=2.9 \%$, Hispanic/Latino $=10.5 \%$, White non-Hispanic $=55.5 \%$

Chicago, IL

$S B(n=134)$ - White $74(55.2 \%)$,

Hispanic 37 (27.6\%), Black 19

(14.2\%), Other 4 (3\%)

Included Spanish speakers.

Cook County (Chicago, IL), $N=$ 5,211,263;

White alone $=65.6 \%$; Black $=$

$24.0 \%$; American Indian $=0.7 \%$;

Asian $=7.7 \%$; Pacific Islander $=$

$0.1 \%$; Two or more races $=1.9 \%$;

Hispanic/Latino $=25.5 \%$; White non-Hispanic $=42.3 \%$

Birmingham, AL

White 116 (73\%), Black 32 (20\%), Hispanic 4 (2.5\%)

English speakers only. Jefferson County (Birmingham, AL), $N=659,197$;

White $=53.2 \%$, Black $=43.4 \%$, American Indian $=0.3 \%$, Asian $=$ $1.7 \%$, Pacific Islander $=0.1 \%$, Two or more races $=1.2 \%$, Hispanic/Latino $=3.9 \%$, White non-Hispanic $=49.9 \%$

Birmingham, AL; Atlanta, GA White 38 (54.2\%), Black 23 (32.8\%), Hispanic 5 (7.2\%), Asian 2 (2.9\%), Not Reported 2 (2.9\%)

No language information provided. Jefferson County (Birmingham, AL), $N=659,197$;

White $=53.2 \%$, Black $=43.4 \%$, American Indian $=0.3 \%$, Asian $=1.7 \%$, Pacific Islander $=0.1 \%$, Two or more races $=1.2 \%$, Hispanic/Latino $=3.9 \%$, White nonHispanic $=49.9 \%$

Fulton County (Atlanta, GA), $N=$ 1,041,423;

White $=45.8 \%$, Black $=44.6 \%$, American Indian $=0.3 \%$, Asian $=$ $7.2 \%$, Pacific Islander $=0.1 \%$, Two or more races $=2.1 \%$, Hispanic/Latino $=7.3 \%$, White non-Hispanic $=39.8 \%$; 
Table 1, continued

\begin{tabular}{|c|c|}
\hline $\begin{array}{l}\text { Author (year); } \\
\text { Sample } N \text { ( } n \text { by } \\
\text { groups) age }\end{array}$ & Design/purpose/ instruments \\
\hline $\begin{array}{l}\text { Smith et al. [44]; } \\
\text { SB } N=210 ; \\
\text { Ages } 4-13 \text { yrs. }\end{array}$ & $\begin{array}{l}\text { Cross-sectional design to identify } \\
\text { effective strategies for managing } \\
\text { urinary and bowel complications } \\
\text { resulting from spina bifida. } \\
\text { To examine the relationship } \\
\text { between parent and child-self } \\
\text { reports using both a generic QOL } \\
\text { instrument (i.e., PedsQL 4.0) and } \\
\text { SB-specific QOL instrument (i.e., } \\
\text { SB-HRQOL). }\end{array}$ \\
\hline
\end{tabular}
SB-HRQOL).

Wasserman and Holmbeck [41], SB $N=95$; Ages 8-15 yrs.

Szymanski et al. [46]; SB $N=298$; Ages 8-17 yrs

Driscoll et

al. [42];

SB $N=140$ dyads (MMC $n=123$, other SB $n=17$ )

Ages 8-19 yrs. mine distinct profiles of cognitive functioning and to examine predictors and outcomes of such profiles using a Ward's three-cluster solution.

To examine the relationship between cluster membership and parent and child-self reports using a generic QOL instrument (i.e., PedsQL 4.0).

Cross-sectional design to determine the impact of urinary and fecal incontinence in children and adolescents with SB using a newly designed SB-specific QOL instrument (i.e., QUALAS) that incorporated the individual's perception. The study also considered developmental delay.

Results

Based on individual and parent reports, no significant associations were found between continence status and QOL measures. However, interviews with the individuals reflected how managing continence at home and school more broadly affects QOL.

Consistent reporting was observed between parent and child self-reports for the both generic and SB-specific QOL instruments (PedsQL 4.0, SB-HRQOL).

SB-specific QOL instrument incorporated the individual's perception of QOL via parent-report by asking questions related to personal relationships, internal beliefs, independence, and future goals.

Cross-sectional design to examine Based on individual and parent reports. neuropsychological performance Cluster membership significantly predicted among children with SB to deter- child reported physical QOL (as well as independence, academic success, parent expectations for the future). SES, lesion level, and seizure history significantly predicted group membership into a cluster of below average, average, and above average groups. These findings suggest that various cognitive profiles exist among children with $\mathrm{SB}$, and these profiles are mediated by SES, lesion level, and seizure history.

Consistent reporting of QOL was observed between parent and child self-reports.

Based on individual self-reports, fecal incontinence is negatively associated with HRQOL in individuals with SB. Urinary incontinence was negatively associated with HRQOL in an age-dependent fashion. HRQOL was lower with increasing amounts of urinary incontinence. QUALAS appears to be an appropriate instrument for assessing QOL in children and adolescents with $\mathrm{SB}$

QOL instrument incorporated the individual's perception of QOL by asking questions related to personal relationships, internal beliefs, and independence.

Based on parent reports, increased levels of parent personal distress, general parenting stress, and SB-specific parenting stress were associated with decreased levels of HRQOL as reported by the individuals with SB. Of these parent factors, SB-specific parenting stress exhibited the strongest association between youth report of QOL and parent proxy report of youth QOL. Additionally, IQ was not associated with parent or child QOL.
Location/demographics/ language information/ county demographics* Los Angeles, CA (site with Spanish speakers); Portland, OR; Birmingham, AL; Seattle, WA No racelethnicity information provided. Included Spanish speakers Los Angeles County (Los Angeles, CA), $N=10,163,507$;

White $=70.9 \%$, Black $=9.0 \%$, American Indian $=1.4 \%$, Asian $=$ $15.3 \%$, Pacific Islander $=0.4 \%$, Two or more races $=3.0 \%$, Hispanic/Latino $=48.6 \%$, White non-Hispanic $=26.2 \%$

Chicago, IL

White 52 (55\%), Non-White 43 (45\%)

Included Spanish speakers

Cook County (Chicago, IL), $N=$ $5,211,263$

White alone $=65.6 \%$, Black $=$ $24.0 \%$, American Indian $=0.7 \%$, Asian $=7.7 \%$, Pacific Islander $=$ $0.1 \%$, Two or more races $=1.9 \%$, Hispanic/Latino $=25.5 \%$, White non-Hispanic $=42.3 \%$

Indianapolis, IN White 226 (76.2\%), Hispanic 29 (9.7\%), Blacks 10 (3.4\%), Asian 8 (2.7\%), Multiethnic 20 (6.7\%)

Excluded Spanish speakers.

Marion County (Indianapolis, IN), $N=950,082$.

White alone $=64.6 \%$, Black $=$ $28.6 \%$, American Indian $=0.5 \%$, Asian $=3.4 \%$, Pacific Islander $=$ $0.1 \%$, Two or more races $=2.9 \%$, Hispanic/Latino $=10.5 \%$, White non-Hispanic $=55.5 \%$

State-wide recruitment in Illinois SB Youth $N=140$ - White 74 (52.9\%), Hispanic 39 (27.9\%), Black 19 (13.6\%), Asian $2(1.4 \%)$, Biracial 6 (4.3\%) Other $0(0 \%)$ Included Spanish speakers * State of Illinois, (Chicago, IL), $N=12,802,023$;

White alone $=77.1 \%$; Black $=$ 14.6\%; American Indian $=0.6 \%$; Asian $=5.7 \%$; Pacific Islander $=$ $0.1 \%$; Two or more races $=2.0 \%$; Hispanic/Latino $=17.3 \%$; White non-Hispanic $=61.3 \%$ 
cific) Pediatric Quality of Life Inventory (PedsQL 4.0) emerged as the instrument most commonly used $(n=$ 11). Seven other QOL instruments, namely the Spina Bifida Health-Related QOL survey (SB-HRQOL; $n=$ 3), the Child Health and Illness Profile - Adolescents Edition survey (CHIP-AE; $n=1$ ), the Child Health Questionnaire - Parent Form 50 survey (CHQ-PF50; $n=1$ ), the Fecal Incontinence and Constipation Quality of Life survey (FICQOL; $n=1$ ), the Glasgow Children's Benefit Inventory (GCBI; $n=1)$, the Health Utilities Index Mark 3 (HUI3; $n=1$ ), and the Quality of Life Assessment in Spina Bifida (QUALAS; $n=$ 1 ), were also used, but less frequently. Although the majority of studies used solely generic assessments of QOL ( $n=13 / 18 ; 72 \%$ ) [30-42], two articles reported the use of both a generic and a SB-specific QOL measure (i.e., PedsQL 4.0 and SB-HRQOL, $n=$ $2 / 18 ; 11 \%)[43,44]$, and three studies $(n=3 / 18$; $17 \%$ ) documented QOL entirely utilizing validated instruments specific to SB (SB-HRQOL, FICQOL, and QUALAS) $[13,45,46]$. Regarding risk of bias, incomplete outcomes data were noted in eight of the 18 studies $[33,34,37-40,42,45]$. Neither selective reporting nor reporting bias was noted among the publications. Random sequence generation, allocation concealment, blinding of participants and personnel, or blinding of outcome assessment was not applicable in the included studies; hence, they were cross-sectional or nonrandomized prospective cohort studies.

\subsection{Social determinant of health: Language as cultural context}

Of the 18 articles, a subset of $12(67 \%)$ articles reported demographic information related to race/ethnicity. Within the 12 articles that reported this demographic information, only seven articles included Hispanics/Latinos. Of these seven, a single study reported $68.2 \%$ of its participants as being Hispanics/Latinos [35]; however, the remaining studies reported a smaller proportion of Hispanics/Latinos (range $=2.5-$ $30 \%$ ) as participants.

The majority of the QOL studies excluded or did not mention including Spanish-speaking individuals. Of the six (33\%) studies that reported inclusion of Spanish-speaking individuals, all used a Spanish version of the generic PedsQL 4.0, and two collaborating studies used an additional SB-HRQOL instrument that the authors reported to have translated at their institutions to complement the generic measure [42,44]. Also, along with these studies, a third reported having a bilingual research assistant [42] to aid with the consent and administration of the surveys to Spanish-speaking parents. No other type of assistance for Spanishspeaking families was described in the remaining publications.

\subsection{Social determinant of health: Health care services}

QOL instruments were also used to examine the influence of accessed surgical services and/or extant mental health on QOL among children and adolescents with SB. Three studies described exploring the influence of surgical procedures such as: the antegrade continence enema procedure (ACE) [45], urinary and fecal incontinence reconstruction surgery [37], reconstructive urological surgery such as augmentation with or without a Mitrofanoff procedure and ACE, and bladder neck reconstruction on QOL [30]. Generic QOL instruments (PedsQL 4.0 and GCBI) were used for two of these three studies. Along with the FICQOL [45], these SB-specific and generic instruments demonstrated changes in QOL following surgery, with post-surgical improvements in HRQOL noted in all three studies. Additionally, since mental health has been reported as a mediator of QOL among children and adolescents [47], two studies incorporated an assessment of mental health (i.e., depression and anxiety) using either the Beck Depression Inventory (BDI) or a subdomain of the FICQOL survey. Flanagan et al. [34] observed that children and adolescents with SB tended to report lower QOL and increased depressive symptoms in comparison to peers with spinal cord injury (SCI) as measured by the BDI. Additionally, Ok et al. [45] reported an overall improvement of QOL and decreased depressive symptoms after the ACE procedure was performed, using the FICQOL.

Lastly, two studies reported on cognitive function outcomes with regard to QOL. In a study by Wasserman and Holmbeck [41], cognitive ability was assessed using the Wechsler Abbreviated Scale of Intelligence (WASI) and subtests from the Wide Range Achievement Tests 3 (WRAT3), the Cognitive Assessment System (CAS), the Delis Kaplan Executive Function System (D-KEFS), the Test of Everyday Attention for Children (TEA-Ch), the Diagnostic Analysis of Nonverbal Accuracy 2 (DANVA2), and the Comprehensive Assessment of Spoken Language (CASL). The results from this study revealed qualitatively different cognitive profiles among children with SB, which ultimately related to physical QOL. Driscoll et al. [42] assessed 
cognitive ability using the WASI but did not observe a significant relation to self-reported or proxy-report HRQOL scores measured on the generic PedsQL 4.0. This study reported on parenting stress as consistently being the most strongly associated with parent proxyreport of youth HRQOL.

\section{Discussion}

Our findings document that individuals living with SB face many factors that negatively impact their reported QOL; these challenges, as well as the QOL instruments used to assess them, are wide-ranging. Along with physical domains, a number of additional factors were associated with lower HRQOL scores; the investigation and understanding of these factors as a basis to develop interventions designed to improve QOL emerged as an important pursuit. Consistent with previous reviews of HRQOL in patients with SB $[26,48]$, our findings demonstrated that most studies were cross-sectional in nature and described varying degrees of HRQOL divergence on parent and selfreported scores related to emotional, social, and school domains (Table 1). Moreover, a generic measure of QOL was utilized by the majority of studies ( $n=$ $15 / 18 ; 83 \%$ ), of which two were supplemented with the use of the SB-HRQOL $[43,44]$. Although generic assessment tools allow comparisons of QOL among individuals with various chronic health conditions, their questions are not specific to the SB population. For instance, the physical health domain questions include items regarding "walking" and "running," which are inapplicable to individuals who rely exclusively on a wheelchair for mobility. In contrast, SB-specific QOL instruments ask questions appropriate for individuals with limited mobility and address degrees of bladder and bowel incontinence that greatly impact an individual's perceived QOL [46].

Thus, current research reasoning has evolved into calling for suitable QOL psychometric instruments that are condition-specific and clinically validated assessment tools that also value individuals' perceptions of their HRQOL rather than unduly focusing on physical function $[48,49]$. The SB-specific QOL instruments are more appropriate for individuals with SB because they offer insight into the person's perspective on QOL. Instruments that increasingly incorporate the individual's perception ask relevant questions related to self-esteem, independence, relationships, and bladder/bowel function, which are essential contribu- tors to overall QOL [9]; in part, SB-specific QOL measures aim to quantify QOL through questions regarding embarrassment, lifestyle, and coping behaviors related to self-catheterization as well as incontinence. Conversely, generic QOL instruments fail to address concerns related to common SB symptoms and focus more on questions such as ambulation. Nonetheless, in our review, the generic PedsQL 4.0 questionnaire was found to be the most pervasive instrument used to measure QOL in individuals with SB. By contrast, only a minority of studies documented solely utilizing a SBspecific instrument for assessment $[13,45,46]$.

The role that cultural context, as a SDH, plays in the outcomes related to chronic health conditions has long been identified [16,50-52]. In particular, language plays a key role as a factor associated with outcomes among Hispanics/Latinos [53,54]. Given the fundamental nature of language, we focused on it as a primary SDH. Health disparities research suggests that among some specific racial/ethnic groups, inferior health status may be explained partly by divergent SDH (e.g., social gradients, social exclusion, and changes in the health care system) [55]. It is also acknowledged that in order to properly address gaps in providing care for patients with $\mathrm{SB}$, a national research strategy must be multi-centered, longitudinal, and inclusive of ethnic and socioeconomic diversity [56]. Promisingly, investigators have also begun to recognize existing disparities through the use of the National Spina Bifida Patient Registry (NSBPR) - a partnership between the CDC and SB clinics throughout the US. Schechter et al. [57], established that non-Hispanic blacks were less likely than the other groups to have documented bladder/bowel continence, as also were Hispanics/Latinos less likely than non-Hispanics. The authors went on to state that after controlling for SB-related intrinsic characteristics, significant variations in outcomes associated with age, gender, race/ethnicity, and insurance status remained. They demonstrated that non-Hispanic blacks, Hispanics/Latinos, and those without private insurance had less favorable outcomes. A second NSBPR study recognized that the likelihood of having bladder continence was significantly higher among nonHispanic whites and those with private insurance [58]. A third NSBPR study demonstrated that non-Hispanic white race/ethnicity and private insurance were associated with an increased likelihood of receiving surgical services to treat neurogenic bowel (e.g., ACE procedure) [59].

In the face of these health disparities, the timely and proper study of QOL among the Hispanic/Latino pop- 
ulation, which has the highest SB prevalence [1], is clear. Furthermore, it has long been recognized that measuring outcome indicators based on the differing cultural aspects of well-being - indicators such as QOL - is crucial [15]. In a previous study beyond health measures alone, Chowanadisai et al. [60] found that well-being assessment, such as satisfaction with health care, is significantly lower among Hispanics/Latinos with SB, even after controlling for socioeconomic status. Since the multidimensional conceptual understanding of an individual's own QOL is thought to be profoundly impacted by the intrinsic cultural context $[52,63]$, health professionals are called upon to consider the impact of culture, ethnicity, and immigration status on QOL [61] in an increasingly ethnically diverse society. Additionally, even after adjustment for family income and parental education, language and culture are known to contribute to the disparities in health outcomes found among Hispanics/Latinos [64]. However, even though it has been demonstrated that culturally sensitive interventions improve QOL and outcomes among Hispanic/Latino children with chronic health conditions [65] and it has been further postulated that such an approach may benefit children with SB [60], efforts towards this goal are nascent in SB care [66].

Whereas researchers utilized supplementary assessment measures to investigate additional dimensions (e.g., stress, anxiety, depression, cognitive function) that theoretically could impact QOL [36,41,42,45], in our US-based review where an estimated 55 million Hispanics/Latinos live, we found no study that probed cultural aspects (e.g., language, acculturation, immigration, etc.) as potential modifiers on QOL. In fact, within the largest minority group and the fastest growing US population segment [25], among whom nearly $75 \%$ of individuals speak Spanish at home [25], there is a dearth of research on HRQOL among those living with SB. Of the 18 QOL studies identified, only six (33\%) reported including Spanish-speaking individuals. In these studies, a generic QOL measure was used, at times supplemented with an ad hoc translation of a condition-specific tool. Although some initial steps were taken to address culturally-appropriate care [4244], further work is needed. Despite Congress' previous efforts to safeguard adequate representation of ethnic and racial minorities in federally funded research, our review failed to identify an adequate culturally validated Spanish-language, SB-specific QOL instrument; this goal is especially relevant to parents and to new immigrants providing self-report. As we grow in our understanding of immigration as a SDH and its effects on health and well-being [67], this gap also becomes self-evident.

Of note, the literature reviewed showed no consistent expression to describe people whose ancestry could be traced to the Spanish-speaking regions of Latin America, including the Caribbean, Mexico, Central America, and South America. Moreover, in some instances, even the term "Spanish origin" was used [36]. Such terminology "places narrow and undue emphasis on the European influence of Spanish colonialism" [68]; thus, the root term Latino would have been preferable.

The authors acknowledge several limitations common to systematic reviews. Inherent limitations exist due to the nature of synthesizing large datasets. To reduce some of these limitations, such as selection bias and inclusion of irrelevant validity/reliability results, a very stringent search criteria was implemented by two reviewers who assessed all studies in accordance with PRISMA guidelines. A second limitation of this review may be that as language and culture were not primary outcomes for many of the QOL studies, they may not have reported all relevant information due to journal limitations or the perception of this information as irrelevant. It is possible that studies that did not report any demographic information did in fact include Hispanics/Latinos or had few Hispanics/Latinos in the community. To address this limitation, we provided county census data [29] relevant to each study site to provide a crude contextual proxy of the percentage of Hispanics/Latinos in the primary catchment area where the research was conducted (Table 1). A third limitation arises from the heterogeneity of the populations (e.g., spinal cord injury) included in the reviewed studies. However, to ensure that we appropriately represented individuals with $\mathrm{SB}$, we did not include any studies with relatively few participants with SB (i.e. studies with less than six participants with SB).

Since its inception [69], those evaluating QOL among individuals with SB have done a remarkable job in developing and moving forward this effort and its associated body of literature. However, these gains have not translated equally to all segments of the SB population. As a starting place, we would encourage the SB research community to include Hispanics/Latinos in QOL studies and conduct interviews and questionnaires in their primary language. After this step is taken, other means will be needed to further delve into additional SDH, health beliefs, and health literacy. Future QOL research should not only take preferred lan- 
guage into account, but it should also explore other dimensions keenly relevant to immigrants such as levels of family support, acculturation, adverse childhood experiences, and resilience.

To our knowledge, this is the first study to summarize the US-based QOL literature under a SDH conceptual framework and to identify gaps in the implementation of these efforts among the population most prevalently affected by SB [1]. Recently, in March 2017, the Spina Bifida Association brought together content experts to update the SB-healthcare guidelines [70]. As these guidelines - comprising 25 areas of care delivery - are implemented, fresh momentum towards the employment of HRQOL measures is likely to ensue. Therefore, nationally we now have renewed guidance and have the opportunity to care for all individuals and parents as whole-persons as they transition across the lifespan [13], not only addressing the medical condition but also holistically approaching them in their language and cultural context.

\section{Conclusions}

"Blue marble health" was introduced in 2013 as a policy framework to illustrate trends in the geographic distribution of neglected tropical diseases affecting atrisk populations that live not only in low-income countries but also in pockets of the populace in wealthier nations [71]. Since that time, it has been noted that this framework may also have a role in illustrating the SB paradigm in a high-income nation such as the US [72]. Globally, since 2000 there has been a $49 \%$ increase in immigration [22], thus linguistically-congruous study of QOL has enlarging relevance not only locally, but also abroad. In the US, in spite of Congress's NIH Revitalization Act of 1993, calling for efforts to ensure that minority groups are included in representative numbers in research studies and that Hispanics/ Latinos have the highest reported prevalence of SB, few HRQOL studies have documented inclusion of this population. According to the most recent US Census forecasts on immigration and birth, this demographic group will increase from $17.4 \%$ to $28.6 \%$ of the total population in the approaching decades; hence, future research should include cultural context as a component in the study of the HRQOL construct and support the utilization of validated Spanish-language measures for its investigation.

\section{Acknowledgments}

The authors thank Dr. Monica Gramatges in the De- partment of Pediatrics, Hematology/Oncology section at Texas Children's Hospital and Dr. Robert G. Voigt, Chief of the Meyer Center for Developmental Pediatrics, Texas Children's Hospital for their support of this work. We also thank Dr. B. Lee Ligon of the Department of Pediatrics, Center for Research, Innovation and Scholarship, Baylor College of Medicine, for editorial assistance. Additionally, we are deeply grateful for the countless individuals and their family members who participated in the research reviewed, without whom progress in the field would not be possible.

\section{Conflict of interest}

The authors declare that they have no conflicts of interest.

\section{References}

[1] Williams J, Mai CT, Mulinare J, Isenburg J, Flood TJ Ethen M, et al. Updated estimates of neural tube defects prevented by mandatory folic Acid fortification - United States, 1995-2011. MMWR Morb Mortal Wkly Rep [Internet]. 2015; 64(1): 1-5. Available from: http://www.ncbi.nlm. nih.gov/pubmed/25590678

[2] Parker SE, Mai CT, Canfield MA, Rickard R, Wang Y, Meyer $\mathrm{RE}$, et al. Updated national birth prevalence estimates for selected birth defects in the united states, 2004-2006. Birth Defects Res Part A - Clin Mol Teratol. 2010; 88(12): 1008-1016. doi: 10.1002/bdra.20735.

[3] Ross ME, Mason CE, Finnell RH. Genomic approaches to the assessment of human spina bifida risk. Birth Defects Res. 2017; 109(2): 120-128. doi: 10.1002/bdra.23592.

[4] Agopian AJ, Canfield MA, Olney RS, Lupo PJ, Ramadhani T, Mitchell LE, et al. Spina bifida subtypes and sub-phenotypes by maternal race/ethnicity in the national birth defects prevention study. Am J Med Genet A. 2012; 158A(1): 109-115. doi: 10.1002/ajmg.a.34383.

[5] Kaufman BA, Terbrock A, Winters N, Ito J, Klosterman A, Park TS. Disbanding a multidisciplinary clinic: Effects on the health care of myelomeningocele patients. Pediatr Neurosurg. 1994; 21(1): 36-44. doi: 10.1159/000120812.

[6] Sawin KJ, Liu T, Ward E, Thibadeau J, Schechter MS, Soe MM, et al. The National Spina Bifida Patient Registry: Profile of a large cohort of participants from the first 10 clinics. J Pediatr. 2015; 166(2): 444-450. doi: 10.1016/j.jpeds.2014.09. 039.

[7] Kinsman SL, Doehring MC. The cost of preventable conditions in adults with spina bifida. Eur J Pediatr Surg [Internet]. 1996; 6(Suppl 1): 17-20. Available from: https://proxy.library. upenn.edu:3150/products/ejournals/pdf/10.1055/s-2008-107 1031.pdf\%0A; http://www.ncbi.nlm.nih.gov/pubmed/90088 13; doi: 10.1055/s-2008-1071031.

[8] Dillon CM, Davis BE, Duguay S, Seidel KD, Shurtleff DB. Longevity of patients born with myelomeningocele. Eur J Pediatr Surg. 2000; 10(Suppl 1): 33-34. doi: 10.1055/s-20081072412 . 
[9] Szymanski KM, Misseri R, Whittam B, Raposo S-M, King SJ, Kaefer M, et al. QUAlity of life assessment in spina bifida for adults (QUALAS-A): Development and international validation of a novel health-related quality of life instrument. Qual Life Res. 2015; 24(10): 2355-2364. doi: 10.1007/s11136-0150988-5.

[10] WHOQOL Group. The world health organization quality of life assessment (WHOQOL): Position paper from the world health organization. Soc Sci Med. 1995; 41(10): 1403-1409. doi: 10.1016/0277-9536(95)00112-k.

[11] Wilkinson R, Marmot M. Social determinants of health: The solid facts. World Health Organization. 2003.

[12] Diderichsen F, Evans T, Whitehead M. The social basis of disparities in health. Challenging Inequities in Health: From Ethics to Action. 2001; 1: 12-23.

[13] Sawin KJ, Brei TJ, Buran CF, Fastenau PS. Factors associated with quality of life in adolescents with spina bifida. J Holist Nurs. 2002; 20(3): 279-304. doi: 10.1177/0898010102 02000307.

[14] Healthy People 2020: An Opportunity to Address the Societal Determinants of Health in the United States. [Internet]. Healthy People 2020: An Opportunity to Address the Societal Determinants of Health in the United States. Available from: https://www.healthypeople.gov/2020/topics-objectives/ topic/social-determinants-of-health

[15] Marmot M, Allen J, Goldblatt P, Boyce T, McNeish D, Grady M. Fair society, healthy lives. The Marmot Review. $2010 \mathrm{Feb}$ 14. doi: 10.1016/j.puhe.2012.05.014.

[16] Walker RJ, Williams JS, Egede LE. Influence of race, ethnicity and social determinants of health on diabetes outcomes. Am J Med Sci. 2016; 351(4): 366-373. doi: 10.1016/j.amjms. 2016.01.008.

[17] BrennanRamirez L, Baker E, Metzler M. Promoting health equity a resource to help communities address social determinants of health. BMJ. 2008.

[18] Joyeux L, Engels AC, Russo FM, Jimenez J, Van Mieghem $\mathrm{T}$, De Coppi $\mathrm{P}$, et al. Fetoscopic versus open repair for spina bifida aperta: A systematic review of outcomes. Fetal Diagn Ther. 2016; 39(3): 161-171. doi: 10.1159/000443498.

[19] Marotta M, Fernández-Martín A, Oria M, Fontecha CG, Giné C, Martínez-Ibáñez V, et al. Isolation, characterization, and differentiation of multipotent neural progenitor cells from human cerebrospinal fluid in fetal cystic myelomeningocele. Stem Cell Res. 2017; 22: 33-42. doi: 10.1016/j.scr.2017.05. 003.

[20] Law P. National institutes of health revitalization act of 1993. 42 USC 289 (a)(1). Google Scholar. 2011; 103-143.

[21] United States Department of Health and Human Services. National Institute of Health: Monitoring Adherence to the NIH Policy on the Inclusion of Women and Minorities as Subjects in Clinical Research. Comprehensive Report: Tracking of Clinical Research as Reported in Fiscal Year 2011 and Fiscal Year 2012 [Internet]. 2013; Available from http://www.sciencedirect.com/science/article/pii/B97801227 40657500137.

[22] UN Department of Economic and Social Affairs. International Migration Report 2017: Highlights. New York, NY: United Nations, 2017.

[23] The Annie E. Casey Foundation; Kids Count Data Center Children in immigrant families in the United States [Internet]. Available at: https://datacenter.kidscount.org/data/tables/ 115-children-in-immigrantfamilies?loc=1\&loct=1\#detailed/.

[24] Pew Research Center WD. Use of Spanish declines among Latinos in major U.S. metros [Internet]. http://www.pewre search.org/fact-tank/2017/10/31/use-of-spanish-declinesamong-latinos-in-major-u-s-metros/. [cited 2018 Sep 10]. Available from: http://www.pewresearch.org/fact-tank/2017/ 10/31/use-of-spanish-declines-among-latinos-in-major-u-smetros.

[25] Bureau of the Census UD of C. US Census Bureau projections show a slower growing, older, more diverse nation a half century from now. [Internet]. December 12, 2012. [cited 2018 Mar 9]. Available from: https://www.census.gov/newsroom/ releases/archives/population/cb12-243.html.

[26] Bakaniene I, Prasauskiene A, Vaiciene-Magistris N. Healthrelated quality of life in children with myelomeningocele: A systematic review of the literature. Child Care Health Dev. 2016; 42(5): 625-643. doi: 10.1111/cch.12367.

[27] Moher D, Liberati A, Tetzlaff J, Altman DG, Altman D, Antes $\mathrm{G}$, et al. Preferred reporting items for systematic reviews and meta-analyses: The PRISMA statement. PLoS Med. 2009; 6(7): e1000097. doi: 10.1371/journal.pmed.1000097.

[28] McGibbon A, Eady A, Marks S. PDQ evidence-based principles and practices. Hamilton: BC Decker. 1999.

[29] US Census Bureau. State and County Quick Facts (v2017). [Internet]. [cited 2018 Sep 1]. Available from: https://www. census.gov/quickfacts/fact/table/US/HSG010217

[30] Parekh AD, Trusler LA, Pietsch JB, Byrne DW, DeMarco RT, Pope JC, et al. Prospective, longitudinal evaluation of health related quality of life in the pediatric spina bifida population undergoing reconstructive urological surgery. J Urol. 2006; 176(4 Pt 2): 1878-1882. doi: 10.1016/S0022-5347(06) 006203.

[31] Abresch RT, McDonald DA, Widman LM, McGinnis K, Hickey KJ. Impact of spinal cord dysfunction and obesity on the health-related quality of life of children and adolescents. J Spinal Cord Med. 2007; 30(Suppl 1): S112-S118. doi: 10.1080/10790268.2007.11754614.

[32] Danielsson AJ, Bartonek Å, Levey E, McHale K, Sponseller $\mathrm{P}$, Saraste H. Associations between orthopaedic findings, ambulation and health-related quality of life in children with myelomeningocele. J Child Orthop. 2008; 2(1): 45-54. doi: 10.1007/s11832-007-0069-6.

[33] Dodson JL, Furth SL, Hsiao C-J, Diener-West M, Levey EB, Wu AW, et al. Health related quality of life in adolescents with abnormal bladder function: An assessment using the Child Health and Illness Profile-Adolescent Edition. J Urol. 2008; 180(4 Suppl): 1846-1851; discussion 1851. doi: 10.1016/j. juro.2008.03.121.

[34] Flanagan A, Gorzkowski M, Altiok H, Hassani S, Ahn KW. Activity level, functional health, and quality of life of children with myelomeningocele as perceived by parents. Clin Orthop Relat Res. 2011; 469(5): 1230-1235. doi: 10.1007/s11999010-1651-7.

[35] Ramachandra P, Palazzi KL, Skalsky AJ, Marietti S, Chiang G. Shunted hydrocephalus has a significant impact on quality of life in children with spina bifida. PM R. 2013; 5(10): 825831. doi: 10.1016/j.pmrj.2013.05.011.

[36] Flanagan A, Kelly EH, Vogel LC. Psychosocial outcomes of children and adolescents with early-onset spinal cord injury and those with spina bifida. Pediatr Phys Ther. 2013; 25(4): 452-459. doi: 10.1097/PEP.0b013e3182a5d35c.

[37] Strine AC, Misseri R, Szymanski KM, Kaefer M, Rhee AC, Hillier K, et al. Assessing health related benefit after reconstruction for urinary and fecal incontinence in children: A parental perspective. J Urol. 2015; 193(6): 2073-2078. doi: 10.1016/j.juro.2014.12.089.

[38] Murray CB, Holmbeck GN, Ros AM, Flores DM, Mir SA, 
Varni JW. A longitudinal examination of health-related quality of life in children and adolescents with spina bifida. J Pediatr Psychol. 2015; 40(4): 419-430. doi: 10.1093/jpepsy/ jsu098.

[39] Rocque BG, Bishop ER, Scogin MA, Hopson BD, Arynchyna AA, Boddiford CJ, et al. Assessing health-related quality of life in children with spina bifida. J Neurosurg Pediatr. 2015; 15(2): 144-149. doi: 10.3171/2014.10.PEDS1441.

[40] Shapiro DR, Malone LA. Quality of life and psychological affect related to sport participation in children and youth athletes with physical disabilities: A parent and athlete perspective. Disabil Health J. 2016; 9(3): 385-391. doi: 10.1016/j. dhjo.2015.11.007.

[41] Wasserman RM, Holmbeck GN. Profiles of neuropsychological functioning in children and adolescents with spina bifida: Associations with biopsychosocial predictors and functional outcomes. J Int Neuropsychol Soc. 2016; 22(8): 804-815. doi: 10.1017/S1355617716000680.

[42] Driscoll CFB, Buscemi J, Holmbeck GN. Parental distress and stress in association with health-related quality of life in youth with spina bifida: A longitudinal study. J Dev Behav Pediatr. 2018; 39(9): 744-753. doi: 10.1097/DBP.0000000000 000603.

[43] Freeman KA, Smith K, Adams E, Mizokawa S, Neville-Jan A. Is continence status associated with quality of life in young children with spina bifida? J Pediatr Rehabil Med. 2013; 6(4): 215-223. doi: 10.3233/PRM-140263.

[44] Smith K, Neville-Jan A, Freeman KA, Adams E, Mizokawa $\mathrm{S}$, Dudgeon BJ, et al. The effectiveness of bowel and bladder interventions in children with spina bifida. Dev Med Child Neurol. 2016; 58(9): 979-988. doi: 10.1111/dmcn.13095.

[45] Ok JH, Kurzrock EA. Objective measurement of quality of life changes after ACE Malone using the FICQOL survey. J Pediatr Urol. 2011; 7(3): 389-393. doi: 10.1016/j.jpurol.2011. 02.012 .

[46] Szymanski KM, Cain MP, Whittam B, Kaefer M, Rink RC, Misseri R. Incontinence affects health-related quality of life in children and adolescents with spina bifida. J Pediatr Urol. 2018; 14(3): 279.e1-279.e8. doi: 10.1016/j.jpurol.2018.02. 021.

[47] Spitzer RL, Kroenke K, Linzer M, Hahn SR, Williams JB, deGruy FV, et al. Health-related quality of life in primary care patients with mental disorders. Results From the PRIMEMD 1000 Study. JAMA. 1995; 274(19): 1511-1517. doi: 10. 1001/jama.1995.03530190025030.

[48] Sawin KJ, Bellin MH. Quality of life in individuals with spina bifida: A research update. Dev Disabil Res Rev. 2010; 16(1): 47-59. doi: 10.1002/ddrr.96.

[49] Waters E, Davis E, Ronen GM, Rosenbaum P, Livingston M, Saigal S. Quality of life instruments for children and adolescents with neurodisabilities: How to choose the appropriate instrument. Dev Med Child Neurol. 2009; 51(8): 660-669. doi: 10.1111/j.1469-8749.2009.03324.x.

[50] Marmot M. Social determinants of health inequalities. Lancet [Internet]. 2005; 365(9464): 1099-1104. Available from: http://www.ncbi.nlm.nih.gov/pubmed/15781105. doi: 10.1016/S0140-6736(05)71146-6.

[51] Larson K, Russ SA, Crall JJ, Halfon N. Influence of multiple social risks on children's health. Pediatrics [Internet] 2008; 121(2): 337-344. Available from: http://www.ncbi.nlm. nih.gov/pubmed/18245425. doi: 10.1542/peds.2007-0447.

[52] Victorino CC, Gauthier AH. The social determinants of child health: Variations across health outcomes - A population- based cross-sectional analysis. BMC Pediatr. 2009; 17(9): 53. doi: 10.1186/1471-2431-9-53.

[53] Keyes KM, Martins SS, Hatzenbuehler ML, Blanco C, Bates LM, Hasin DS. Mental health service utilization for psychiatric disorders among Latinos living in the United States: The role of ethnic subgroup, ethnic identity, and language/social preferences. Soc Psychiatry Psychiatr Epidemiol. 2012; 47(3): 383-394. doi: 10.1007/s00127-0100323-y.

[54] Larimer KA, Gulanick M, Penckofer S. Understanding determinants of cardiovascular health in a Mexican American community. Health Promot Pract. 2017; 18(4): 534-544. doi: 10.1177/1524839917698095.

[55] Flores G, Lin H. Trends in racial/ethnic disparities in medical and oral health, access to care, and use of services in US children: Has anything changed over the years? Int J Equity Health. 2013; 12: 10. doi: 10.1186/1475-9276-12-10.

[56] Sawin KJ, Betz CL, Linroth R. Gaps and opportunities: An agenda for further research, services, and program development in spina bifida. Pediatr Clin North Am. 2010; 57(4): 1041-1057. doi: 10.1016/j.pcl.2010.07.020.

[57] Schechter MS, Liu T, Soe M, Swanson M, Ward E, Thibadeau $\mathrm{J}$. Sociodemographic attributes and spina bifida outcomes. Pediatrics. 2015; 135(4): e957-e964. doi: 10.1542/peds.20142576.

[58] Liu T, Ouyang L, Thibadeau J, Wiener JS, Routh JC, Castillo $\mathrm{H}$, et al. Longitudinal study of bladder continence in patients with spina bifida in the national spina bifida patient registry. J Urol. 2017; 199(3): 837-843. pii: S0022-5347(17)77886-X. doi: 10.1016/j.juro.2017.11.048.

[59] Routh JC, Joseph DB, Liu T, Schechter MS, Thibadeau JK, Chad Wallis M, et al. Variation in surgical management of neurogenic bowel among centers participating in national spina bifida patient registry. J Pediatr Rehabil Med. 2017; 10(3-4): 303-312. doi: 10.3233/PRM-170460.

[60] Chowanadisai M, de la Rosa Perez DL, Weitzenkamp DA, Wilcox DT, Clayton GH, Wilson PE. The role of ethnicity and culture on functional status in children with spina bifida. J Pediatr Rehabil Med. 2013; 6(4): 205-213. doi: 10.3233/PRM140259.

[61] Kagawa-Singer M, Padilla GV, Ashing-Giwa K. Healthrelated quality of life and culture. Semin Oncol Nurs. 2010; 26(1): 59-67. doi: 10.1016/j.soncn.2009.11.008.

[62] Shumaker SA. The international assessment of health-related quality of life: A theoretical perspective. The International Assessment of Health-Related Quality of Life. 1995; 3-10.

[63] Kagawa-Singer M, Chung RC. Toward a new paradigm: A cultural systems approach. In Asian American Mental Health. 2002; 47-66. Springer, Boston, MA. doi: 10.1007/978-14615-0735-2_4.

[64] Flores G, Bauchner H, Feinstein AR, Nguyen US. The impact of ethnicity, family income, and parental education on children's health and use of health services. Am J Public Health. 1999; 89(7): 1066-1071. doi: 10.2105/ajph.89.7.1066.

[65] Mccallum GB, Morris PS, Brown N, Chang AB. Culturespecific programs for children and adults from minority groups who have asthma. Cochrane Database Syst Rev. 2017; 8: CD006580. doi: 10.1002/14651858.CD006580.pub5.

[66] Castillo J, Perez A, Morrison Jacobus M, Bolin RG, Fremion E, Ostermaier K, et al. Latin american-descent families' educational needs: Opportunity for international advocacy and collaboration to promote skin health. J Pediatr Rehabil Med. 2017; 10(S1): S81-S110. doi: 10.3233/PRM-170430.

[67] Castañeda H, Holmes SM, Madrigal DS, Young M-ED, 
Beyeler N, Quesada J. Immigration as a social determinant of health. Annu Rev Public Health. 2015; 36375-92. doi: 10. 1146/annurev-publhealth-032013-182419.

[68] Flores G. Culture and the patient-physician relationship: Achieving cultural competency in health care. J Pediatr. 2000; 136(1): 14-23. doi: 10.1016/S0022-3476(00)90043-X.

[69] Parkin PC, Kirpalani HM, Rosenbaum PL, Fehlings DL, Van Nie A, Willan AR, et al. Development of a health-related quality of life instrument for use in children with spina bifida. Qual Life Res. 1997; 6(2): 123-132. doi: 10.1023/A:1026 486016212 .
[70] Wiener JS, Suson KD, Castillo J, Routh JC, Tanaka S, Liu T, et al. Bowel management and continence in adults with spina bifida: Results from the national spina bifida patient registry 2009-15. J Pediatr Rehabil Med. 2017; 10(3-4): 335-343. doi: 10.3233/PRM-170466.

[71] Hotez PJ, Damania A, Naghavi M. Blue marble health and the global burden of disease study 2013. PLoS Neglected Tropical Diseases. 2016; 10(10): e0004744. doi: 10.1371/journal.pntd. 0004744.

[72] Castillo J, Castillo H. Global health and chronic noncommunicable conditions: Spina bifida care across a worldwide community. J Pediatr Rehabil Med. 2017; 10(3-4): 167168. doi: 10.3233/PRM-170471. 